Article

\title{
Jatrorrhizine Hydrochloride Suppresses RANKL-Induced Osteoclastogenesis and Protects against Wear Particle-Induced Osteolysis
}

\author{
Hui Li, Jing Wang, Qiwen Sun, Gang Chen, Shengnan Sun, Xuemei Ma, Haiwen Qiu, \\ Xuerong Liu, Liangyi Xu and Mei Liu* \\ Jiangsu Key Laboratory for Molecular and Medical Biotechnology, College of Life Sciences, \\ Nanjing Normal University, Nanjing 210023, China; 15050527874@163.com (H.L.); njskywj@163.com (J.W.); \\ sunqiwen123@163.com (Q.S.); m18852005775@163.com (G.C.); alyssia_s@163.com (S.S.); \\ njnumaxuemei@163.com (X.M.); 15895937858@163.com (H.Q.); 13852240843@163.com (X.L.); \\ 15651792179@163.com (L.X.) \\ * Correspondence: liumei@njnu.edu.cn; Tel.: +86-25-8589-1050
}

Received: 23 September 2018; Accepted: 15 November 2018; Published: 21 November 2018

\begin{abstract}
Wear particle-induced aseptic prosthetic loosening is a major complication associated with total joint arthroplasty (TJA). A growing body of evidence suggests that receptor activator of nuclear factor $\mathrm{k}-\mathrm{B}$ ligand (RANKL)-stimulated osteoclastogenesis and bone resorption are responsible for peri-implant loosening. Thus, agents which attenuate excessive osteoclast differentiation and function have been considered to offer therapeutic potential for prolonging the life of TJA implants. Jatrorrhizine hydrochloride $(\mathrm{JH})$, a major protoberberine alkaloid isolated from the traditional Chinese herb Coptis chinensis, has been reported to have antimicrobial, antitumor, and antihypercholesterolemic and neuroprotective activities. However, its effects on osteoclast biology remain unknown. Here, we found that JH inhibited RANKL-induced osteoclast formation and bone resorption in vitro and exerted protection against titanium (Ti) particle-induced osteolysis in vivo. Biochemical analysis demonstrated that JH suppressed RANKL-induced activation of MAPKs (p38 and ERK) which down-regulated the production of NFATc1 and NFATc1-regulated osteoclastic marker genes, such as TRAP, CTR and CTSK. Collectively, our findings suggest that $\mathrm{JH}$ may be a promising anti-osteoclastogenesis agent for treating periprosthetic osteolysis or other osteoclast-related osteolytic diseases.
\end{abstract}

Keywords: jatrorrhizine hydrochloride; osteoclast formation; bone resorption; wear particle-induced osteolysis; MAPK signaling pathway

\section{Introduction}

Total joint arthroplasty (TJA) is an effective procedure for treating end-stage joint diseases such as rheumatoid arthritis and osteoarthritis [1]. However, periprosthetic osteolysis and subsequent aseptic loosening is the leading complication which reduces the lifespan of implants [2,3]. Several strategies have been developed to overcome this problem, including improving the implant designs, perfecting surgical techniques, and searching for better biomaterials. However, these strategies are unlikely to completely eliminate particle generation from implants [4,5]. Despite the precise mechanisms remaining unclear, wear particle-triggered over-activated osteoclastogenesis and extensive bone erosion have been demonstrated to be vital etiologies in periprosthetic osteolysis [6,7]. During the last two decades, many agents have been demonstrated to target osteoclast function and suppress wear debris-stimulated bone resorption, such as bisphosphonates [8,9], erythromycin [10] and denosumab [11]. However, their long-term use could cause severe side effects, including digestive 
system disorders, osteonecrosis, and even some types of malignancy [12-15]. Therefore, it is still of great importance to explore alternative drugs with less side effects to address these problems.

Jatrorrhizine hydrochloride $(\mathrm{JH})$ is one of the major protoberberine alkaloids isolated from the herb C. chinensis, and has been reported to possess some pharmacological properties, such as bacteriostasis [16,17], antioxidation [18], tumor cell growth suppression [19,20], and antidiabetic and antihyperlipidemic activities [21,22]. Nevertheless, to our knowledge, no study has investigated its anti-osteoclastogenic and anti-resorptive actions. In our recently published paper, JH has been demonstrated to inhibit focal bone erosion in collagen-induced arthritis (CIA) rat model [23]. As osteoclast is the sole cell type responsible for bone resorption, therefore, we speculated that $\mathrm{JH}$ may have a potential therapeutic role of anti-osteolytic diseases through inhibiting osteoclastogenesis. We tested this possibility in the current study, using a Ti particle-induced murine calvarial osteolytic model in vivo and RANKL-induced osteoclastogenesis in vitro, and subsequently investigated their underlying mechanisms.

\section{Results}

\subsection{Therapeutic Effect of JH on Ti Particle-Induced Osteolysis In Vivo}

To investigate the possible inhibitory effect of $\mathrm{JH}$ on pathological osteolysis in vivo, a Ti particle-induced murine calvarial osteolytic model was established. Three-dimensional reconstruction data revealed that, compared to the sham group, Ti particles induced severe osteolysis, as evidenced by extensive erosion on the bone surface of the vehicle group (Figure 1A). In contrast, JH significantly and dose-dependently reduced the severity of Ti particle-induced osteolysis. Bone loss in the high-dose JH treated group was markedly less than that in the low-dose group (Figure 1A). Also, our quantitative $\mu \mathrm{CT}$ analysis showed that, compared to the vehicle group, JH treatment significantly increased bone mineral density (BMD) as well as bone volume/tissue volume (BV/TV) (Figure 1B), which further confirmed the reconstruction data.

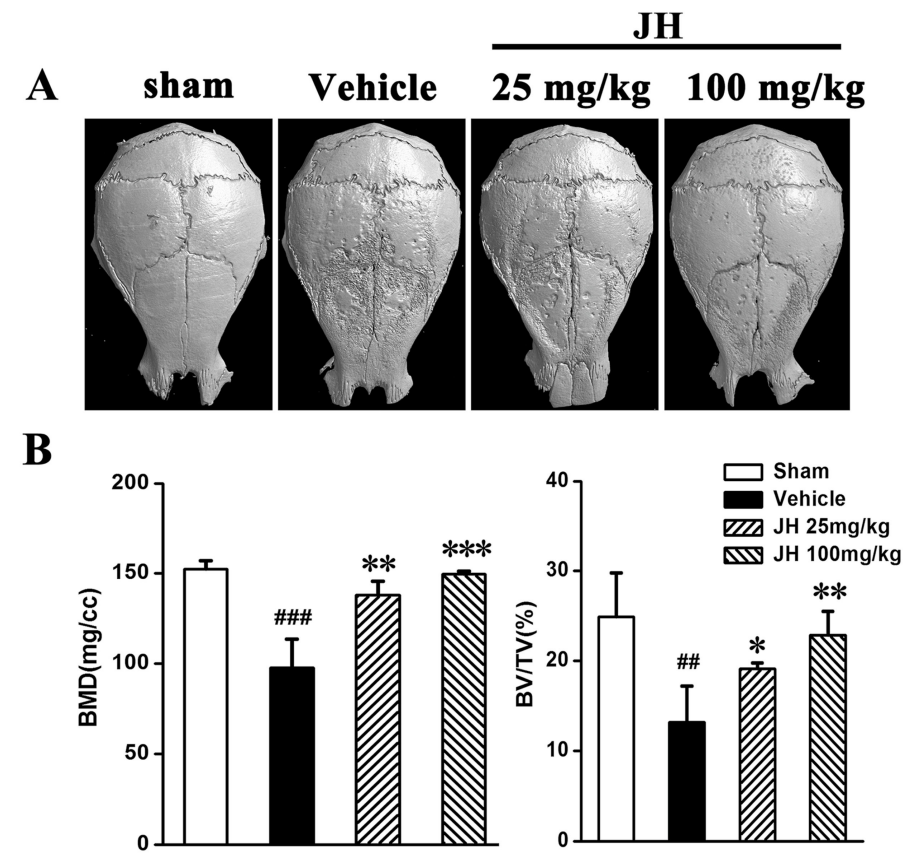

Figure 1. JH inhibits $\mathrm{Ti}$ particle-induced bone destruction in a mouse calvarial model. (A) Representative micro-CT 3D reconstructed images were obtained from different groups. (B) Bone mineral density (BMD) and bone volume/tissue volume (BV/TV) were determined using the CTan program (SkyScan). All the data were shown as mean $\pm \mathrm{SD}$; \# $p<0.01$ and \#\#\# $p<0.001$ versus sham group, ${ }^{*} p<0.05,{ }^{* *} p<0.01$ and ${ }^{* * *} p<0.001$ versus vehicle-treated Ti particle-induced group; $n=7$ per group. 
Histological assessment and histomorphometric analysis further demonstrated the amelioration of Ti particle-induced osteolysis by JH. Hematoxylin and eosin (H\&E) staining showed that bone erosion had clearly occurred in the vehicle group, whereas $\mathrm{JH}$ treatment significantly reduced these osteolytic changes (Figure 2A). This result was confirmed by histomorphometric analysis. As shown in Figure 2B, both low and high doses of JH significantly reduced the erosive surface. Furthermore, tartrate-resistant acid phosphatase (TRAP) staining showed that, in the vehicle-treated group, the number of TRAP ${ }^{+}$osteoclasts mainly distributing along the eroded surface was significantly increased. This increase was effectively decreased by JH treatment (Figure 2C), indicating that the inhibitory effect of $\mathrm{JH}$ on Ti particle-induced bone erosion was associated with the formation and function of osteoclasts.

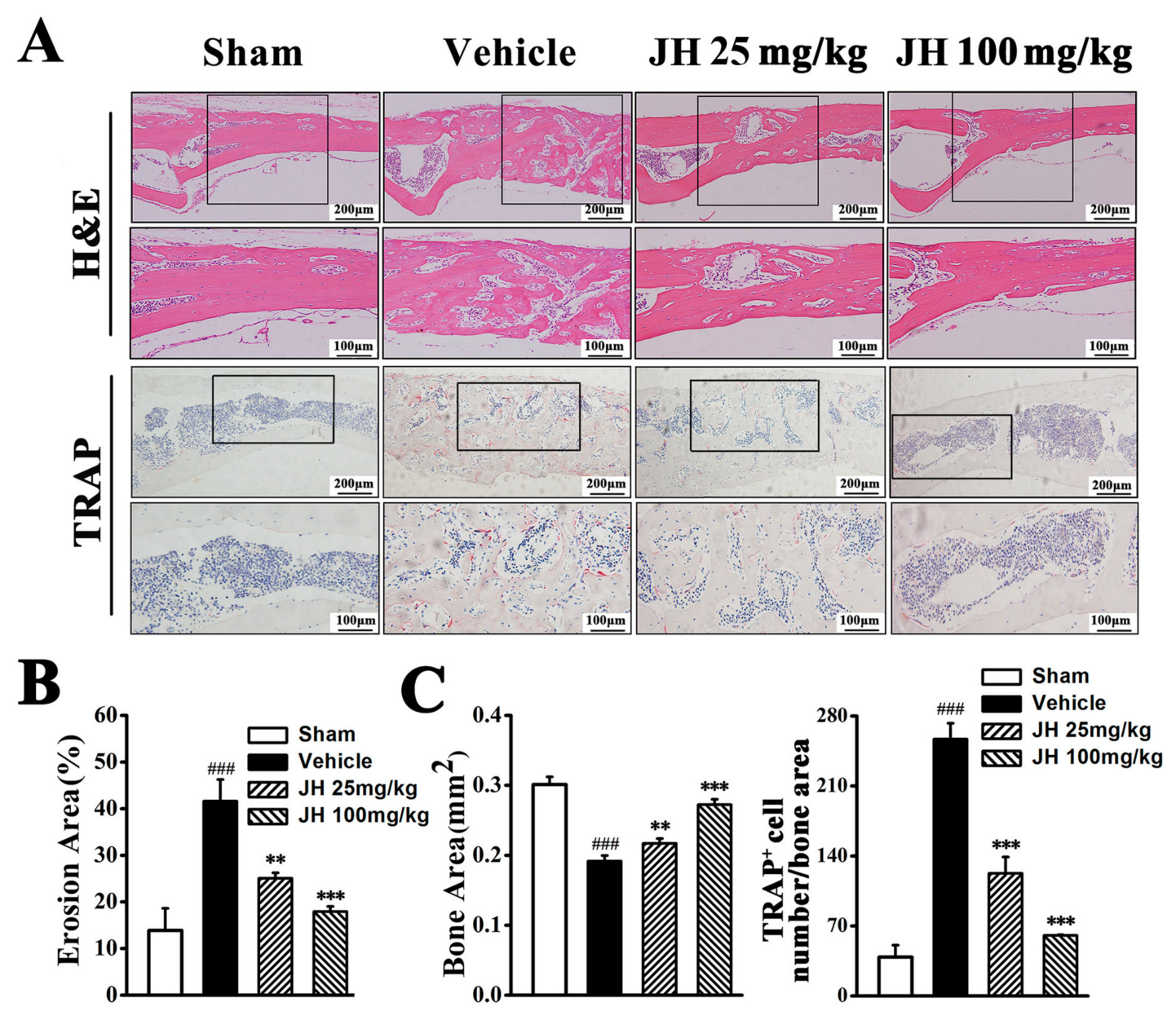

Figure 2. Histological staining and histomorphometric assessment of the inhibitory actions of JH on $\mathrm{Ti}$ particle-induced osteolysis. (A) Representative micrographs staining for H\&E and tartrate-resistant acid phosphatase (TRAP). The regions marked by the black frames were enlarged and shown in the corresponding bottom panels. (B) The percentage of total porosity obtained from the H\&E-stained sections. (C) The bone area $\left(\mathrm{mm}^{2}\right)$ and $\mathrm{TRAP}^{+}$osteoclast number in TRAP-stained sections were calculated and analyzed as described in the Section 4. All data were shown as mean $\pm \mathrm{SD}$; \#\# $p<0.001$ versus sham group, ${ }^{* *} p<0.01$ and ${ }^{* *} p<0.001$ versus vehicle-treated Ti particle-induced group.

In addition to efficiency, safety issues need to be addressed. In this study, JH-treated groups displayed normal behavioral activities and no obvious adverse effects or mortality occurred. Also, we examined the body weight and serum levels of alanine aminotransferase (ALT) and aspartate aminotransferase (AST) in JH treatment groups. As shown in Table 1, there was almost no body change in the JH-treated mice, as compared with the untreated Ti particle-induced mice and the control mice. In addition, JH-treated mice did not show any significant changes in serum ALT and AST levels, 
as compared with the untreated mice, further demonstrating that the present $\mathrm{JH}$ treatment had no significant adverse side effects.

Table 1. Effects of JH on body weight and serum levels of ALT and AST in Ti particle-induced mice.

\begin{tabular}{|c|c|c|c|c|}
\hline Parameters & Control & Vehicle & $\mathrm{JH}\left(25 \mathrm{mg} \cdot \mathrm{kg}^{-1}\right)$ & $\mathrm{JH}\left(100 \mathrm{mg} \cdot \mathrm{kg}^{-1}\right)$ \\
\hline Initial weight (g) & $22.02 \pm 1.30$ & $21.60 \pm 0.61$ & $21.99 \pm 0.57$ & $21.46 \pm 0.41$ \\
\hline Final weight (g) & $22.29 \pm 1.56$ & $22.37 \pm 0.42$ & $22.45 \pm 0.76$ & $22.94 \pm 0.73$ \\
\hline AST $(\mathrm{U} / \mathrm{L})$ & $27.88 \pm 1.80$ & $29.37 \pm 2.48$ & $22.96 \pm 4.47$ & $22.55 \pm 3.31$ \\
\hline $\operatorname{ALT}(\mathrm{U} / \mathrm{L})$ & $9.99 \pm 1.83$ & $8.68 \pm 1.84$ & $8.60 \pm 0.91$ & $9.06 \pm 2.15$ \\
\hline
\end{tabular}

Ti particle-induced mice were administrated with different doses of JH $\left(25 \mathrm{mg} \cdot \mathrm{kg}^{-1}\right.$ and $\left.100 \mathrm{mg} \cdot \mathrm{kg}^{-1}\right)$ or PBS daily for up to 14 days. Data are expressed as mean $\pm \mathrm{SD}(n=7)$. ALT, alanine aminotransferase; AST, aspartate aminotransferase.

\subsection{JH Inhibited RANKL-Induced Osteoclast Formation In Vitro}

To investigate the mechanisms through which JH inhibited Ti particle induced-osteolysis, the effect of $\mathrm{JH}$ on osteoclast formation was determined in vitro. Bone marrow-derived macrophages (BMMs) were treated with different concentrations of JH for 5 days in presence of RANKL. In the control group, numerous TRAP-positive multinucleated osteoclasts were formed. However, JH at the dosages 5-20 $\mu \mathrm{M}$ significantly and dose-dependently inhibited the number and area of osteoclasts (Figure 3A,B). To investigate at which stage JH inhibited osteoclastogenesis, we firstly performed TRAP staining on $\mathrm{d} 0-\mathrm{d} 5$ of RANKL induction to define the different stages of osteoclastogenesis. As shown in Figure 3C, TRAP-positive mononuclear osteoclasts were mainly formed at the first two days of RANKL stimulation. At day 3 of RANKL induction, the multinucleated cells (MNCs) were obviously observed, and the number and size of TRAP ${ }^{+} \mathrm{MNC}$ s were gradually increased with RANKL continuous treatment for $4-5$ days. Thus, JH was added to osteoclast differentiation cultures at day 0 (the day RANKL was added), day 2 (RANKL induction for 2 days) and $12 \mathrm{~h}$ prior to RANKL stimulation. As shown in Figure 3D and 3E, JH treatment on the first two days of RANKL induction (Early Treatment) did not affect osteoclast formation, however, JH treatment $12 \mathrm{~h}$ prior to RANKL induction (Pre-Treatment) modestly but statistically significantly inhibited osteoclastogenesis. This is an interesting observation, and the involved molecular mechanisms remain to be clarified. In addition, $\mathrm{JH}$ treatment on day 3 of the 5 day culture (Late Treatment) modestly reduced the number or area of osteoclasts formed. Consecutive treatment with JH, for example 2 stages (Early and late) or 3 stages (Pre, Early and Late), showed a strong inhibitory effect on osteoclast formation (Figure 3D,E). To exclude the potential cytotoxic effect of JH on BMMs, a cell survival assay was performed. Our results showed that BMM viability or proliferation was not affected by JH treatment, even at concentration as high as $160 \mu \mathrm{M}$. (Figure 3F). To investigate the possible effect of apoptosis on osteoclast formation, an apoptosis assay was performed by flow cytometry. As shown in Figure 3G, concentrations of JH which were effective at inhibiting osteoclastogenesis did not affect apoptosis, indicating the inhibitory action of $\mathrm{JH}$ on osteoclastogenesis was not due to apoptosis. 

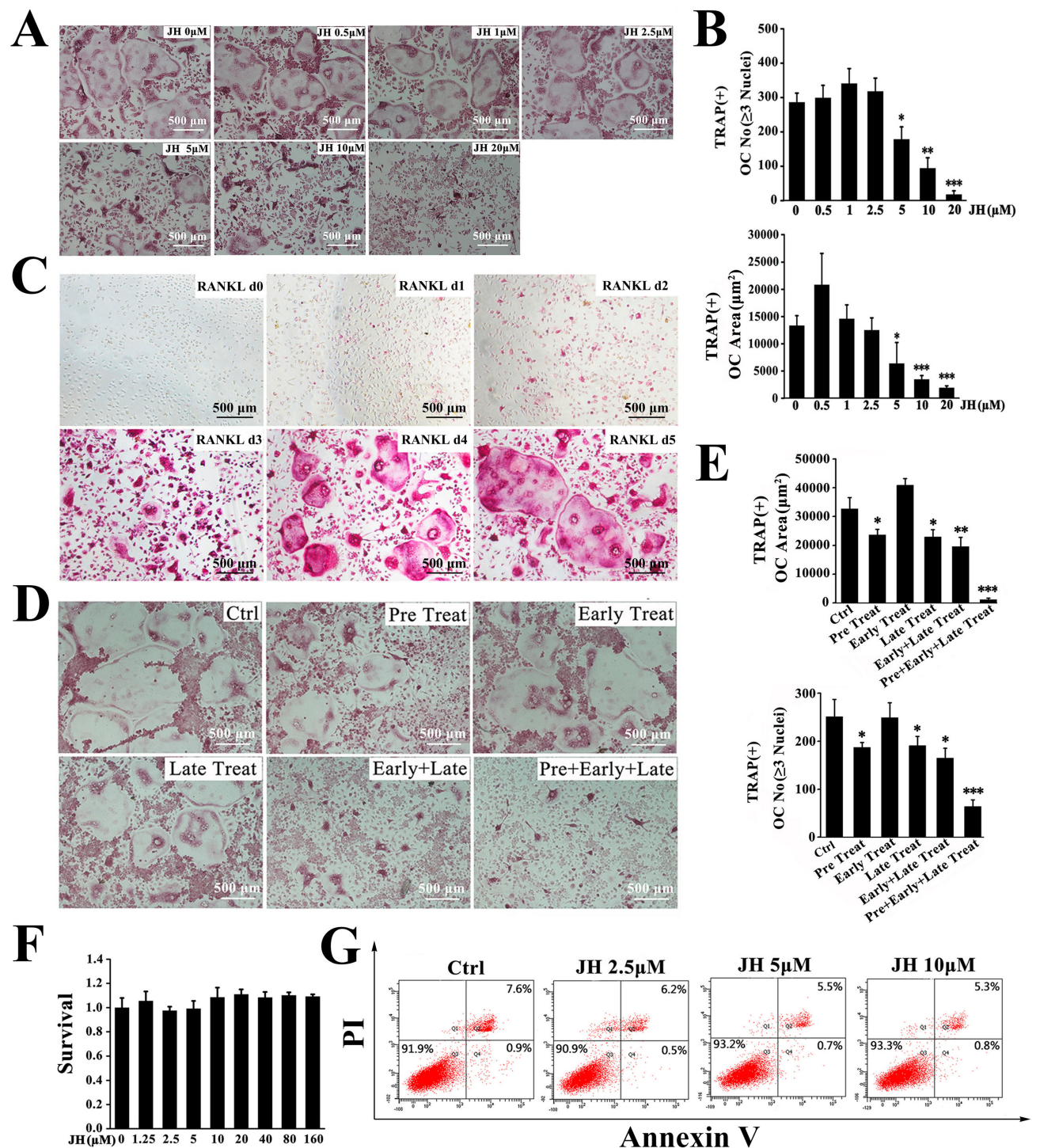

Figure 3. JH inhibits osteoclast formation in vitro. (A) A representative image showing the inhibitory effect of JH on osteoclast formation. BMM cells were treated with different concentrations of $\mathrm{JH}$ in the presence of RANKL (100 ng. $\left.\mathrm{mL}^{-1}\right)$ for 5 days and then TRAP staining was performed. The number and area of $\mathrm{TRAP}^{+}$multinucleated cells with more than three nuclei were shown in (B), $n=3,{ }^{*} p<0.05$, ** $p<0.01$ and ${ }^{* *} p<0.001$ relative to RANKL-treated, JH-untreated controls. OC No: osteoclast number. (C) To define the different stages of osteoclastogenesis, TRAP staining was performed on d0-d5 of RANKL induction (d0, the day RNAKL was added; d1, RANKL induction for 1 day; d2, RANKL induction for 2 days; and so on). (D) BMMs were cultured in the presence of RANKL, and $\mathrm{JH}(5 \mu \mathrm{M})$ was added in different times. At the end of 5 days, TRAP staining was performed. (Ctrl group: RANKL induction for 5 days without JH; Pre Treat group: JH pre-treatment for $12 \mathrm{~h}$ and RANKL induction for 5 days; Early Treat group: Co-treatment with JH and RANKL for 2 days and RANKL continued induction for 3 days; Late Treat group: RANKL induction for 2 days and subsequent co-treatment with JH and RANKL for 3 days; Early + Late Treat group: Co-treatment with JH and RANKL for 5 days; Pre + Early + Late Treat group: JH treatment for $12 \mathrm{~h}$ and subsequent co-treatment with JH and RANKL for 5 days) (E) The area and number of TRAP ${ }^{+}$osteoclasts ( $\geq 3$ nuclei) from (D) were quantified $(n=3)$. All data were shown as mean $\pm \mathrm{SD} ;{ }^{*} p<0.05,{ }^{* *} p<0.01$ and ${ }^{* *} p<0.001$ relative to RANKL-treated, JH-untreated controls. (F) Effect of JH on cell viability was measured using a MTS assay. (G) BMMs were exposed to the indicated concentrations of JH for $24 \mathrm{~h}$, and then cell apoptosis was determined using flow cytometry. 


\subsection{JH Impaired Ostoclastic Bone Resorption and F-Actin Ring Formation In Vitro}

Given that JH can inhibit osteoclast differentiation, we used mature osteoclasts to investigate the effect of $\mathrm{JH}$ on resorptive function. As shown in Figure $4 \mathrm{~A}, \mathrm{~B}$, treating mature osteoclasts with vary concentrations of $\mathrm{JH}$ did not alter the viability of cells, indicating that $\mathrm{JH}$ had no cytotoxic effect on mature osteoclasts. The resorptive pits on the bone slices were scanned by scanning electron microscopy. As expected, mature osteoclasts extensively resorbed bone matrix in the control group, however, JH significantly and dose-dependently attenuated osteoclast-mediated bone resorption (Figure 4C,D).
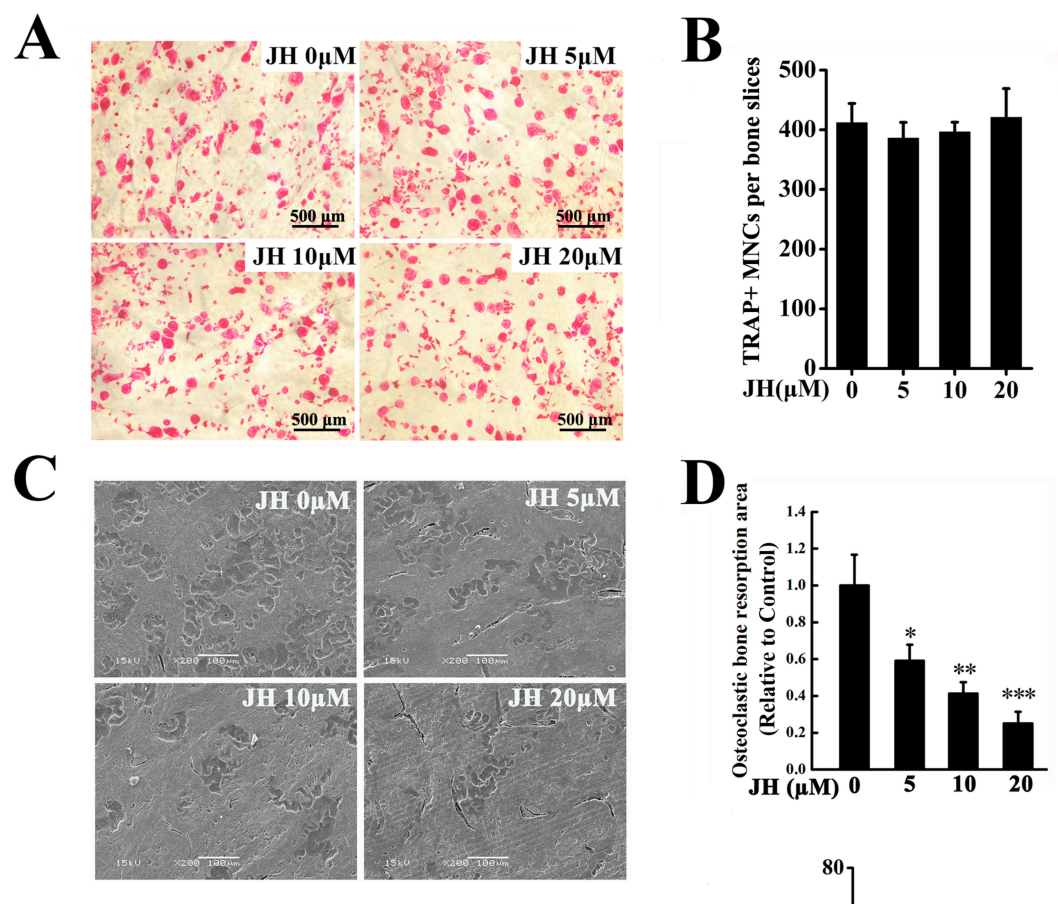

$\mathbf{E}$
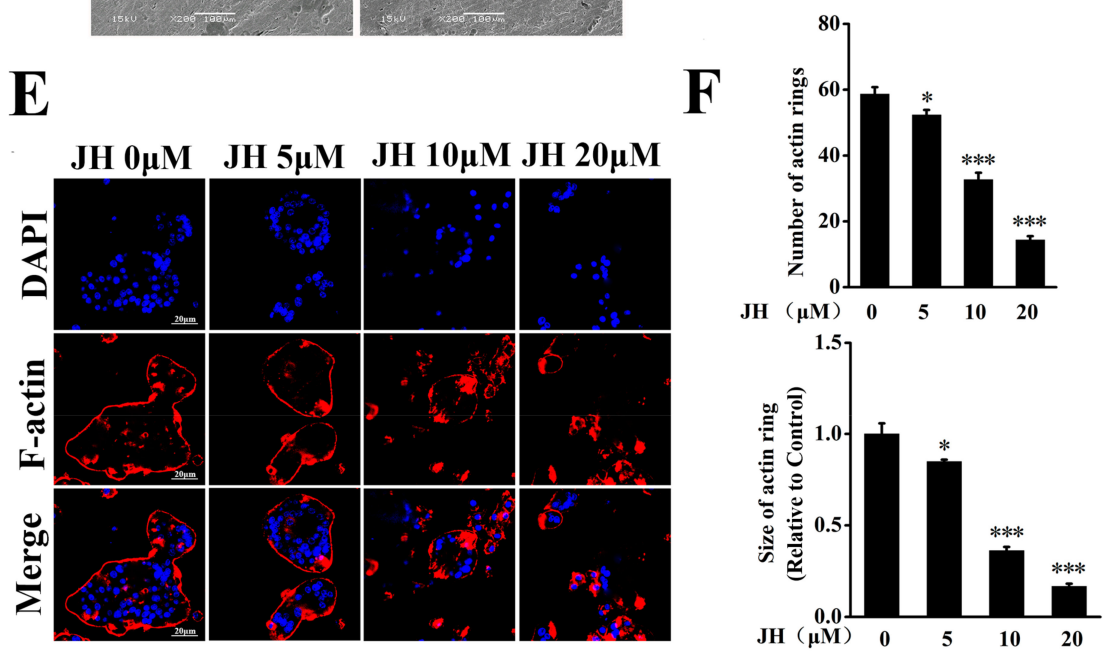

Figure 4. JH inhibits osteoclast-mediated bone resorption. (A) Representative TRAP staining images showing no cytotoxic effect of $\mathrm{JH}$ on mature osteoclasts. (B) The number of $\mathrm{TRAP}^{+}$osteoclasts ( $\geq 3$ nuclei) from (A) were quantified $(n=3)$. (C) Representative scanning electron microscopy (SEM) images showing the inhibitory effect of $\mathrm{JH}$ on osteoclast-mediated bone resorption. (D) Resorption area relative to the total area of bone was measured using ImageJ $(n=3)$. (E) Representative immunofluorescence staining images showing the inhibitory effect of $\mathrm{JH}$ on F-actin ring formation. (F) The number and size of intact actin rings from (E) were quantified $(n=3) .{ }^{*} p<0.05,{ }^{* *} p<0.01$ and *** $p<0.001$ relative to RANKL-treated, JH-untreated controls. 
A well-polarized F-actin ring is well known to be a prerequisite for osteoclastic resorption. To further confirm the effect of $\mathrm{JH}$ on bone resorption, we investigated whether $\mathrm{JH}$ can affect $\mathrm{F}$-actin ring formation. A shown in Figure 4E,F, well-structured F-actin rings were found in the control group. However, JH significantly and dose-dependently blocked F-actin ring formation, as evidenced by the decreased number and size of F-actin rings in JH-treated groups. These data indicated that the inhibitory effect of $\mathrm{JH}$ on osteoclastic resorption could partially be the result of impairing F-actin ring formation.

\subsection{JH Inhibited RANKL-Induced Osteoclast-Specific Gene Expression}

To further evaluate the inhibitory effect of JH on osteoclastogenesis, real-time PCR was performed. As shown in Figure 5, the expressions of osteoclast-specific genes including cathepsin K (CTSK), calcitonin receptor (CTR), TRAP, nuclear factor of activated T-cells cytoplasmic 1 (NFATc1) and c-fos were significantly suppressed by JH at day 5 of culture. These data were consistent with the results of osteoclast formation and bone-resorptive activity assays.
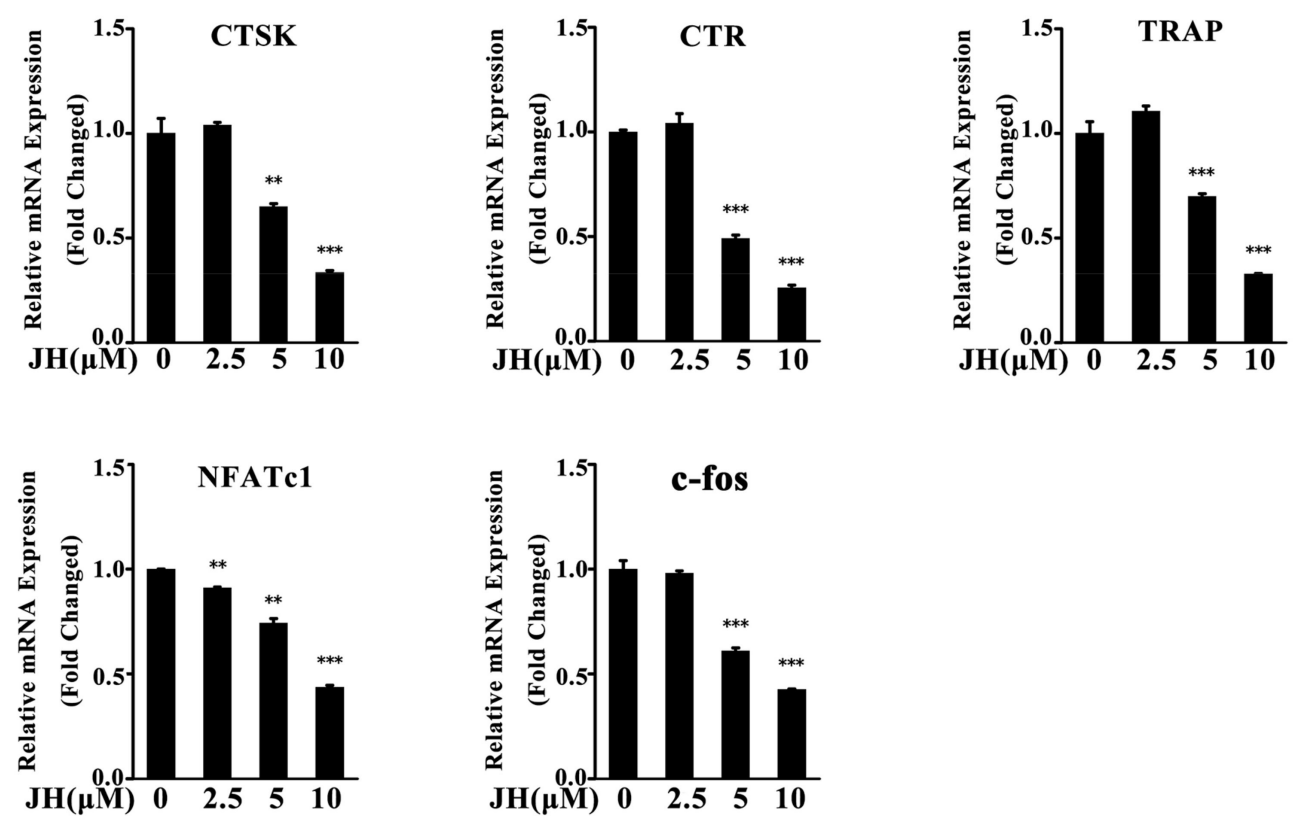

Figure 5. JH suppresses RANKL-induced osteoclast-specific gene expression during osteoclastogenesis. BMMs were treated with RANKL and varying concentrations of JH for 5 days. The levels of osteoclast-specific marker genes were analyzed by real-time PCR, normalized to $\beta$-actin expression $(n=3)$. All data were shown as mean $\pm \mathrm{SD} ;{ }^{* *} p<0.01$ and ${ }^{* *} p<0.001$ relative to RANKL-treated, JH-untreated controls. CTSK: cathepsin K; CTR: calcitonin receptor; TRAP: tartrate-resistant acid phosphatase.

\subsection{JH Inhibited RANKL-Induced MAPK and NFATc1 Activation}

To explore the underlying mechanism through which JH-mediated osteoclast formation, RANKL-induced MAPK and NF- $\mathrm{BB}$ signaling pathways were investigated. As shown in Figure 6A,B, RANKL stimulation significantly increased the phosphorylation of the MAPK family members including extracellular signal-regulated kinase (ERK), c-Jun N-terminal kinase (JNK) and p38 kinase. The increases of p-p38 and p-ERK1/2 were significantly and dose-dependently inhibited by JH treatment. However, JH had no obvious effect on phosphorylation of JNK and degradation of IKBa, indicating that it does not influence the JNK and NF-KB pathways.

Activations of the MAPK (p38 and ERK) pathways subsequently lead to the induction and activation of NFATc1, which has been reported to be a master transcription factor for osteoclast differentiation and function [24]. Thus, we investigated whether JH affected the induction of NFATc1. 
As shown in Figure 6C,D, the protein level of NFATc1 was increased when cells were exposed to RANKL, and JH significantly attenuated this increase. Since c-fos has been reported to bind to the promoter of NFATc1 and thereafter regulate its expression [25], we next examined the protein level of c-fos. As expected, the level of c-fos was significantly decreased by JH treatment, further confirming the inhibitory action of JH on RANKL-induced NFATc1 expression (Figure 6C,D).

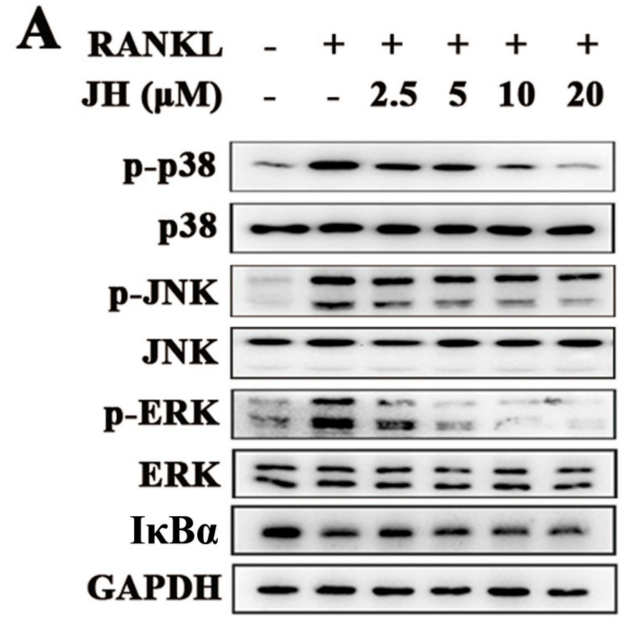

C

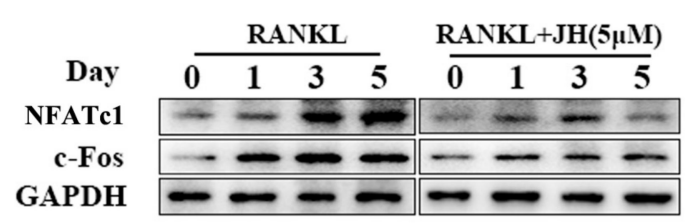

B
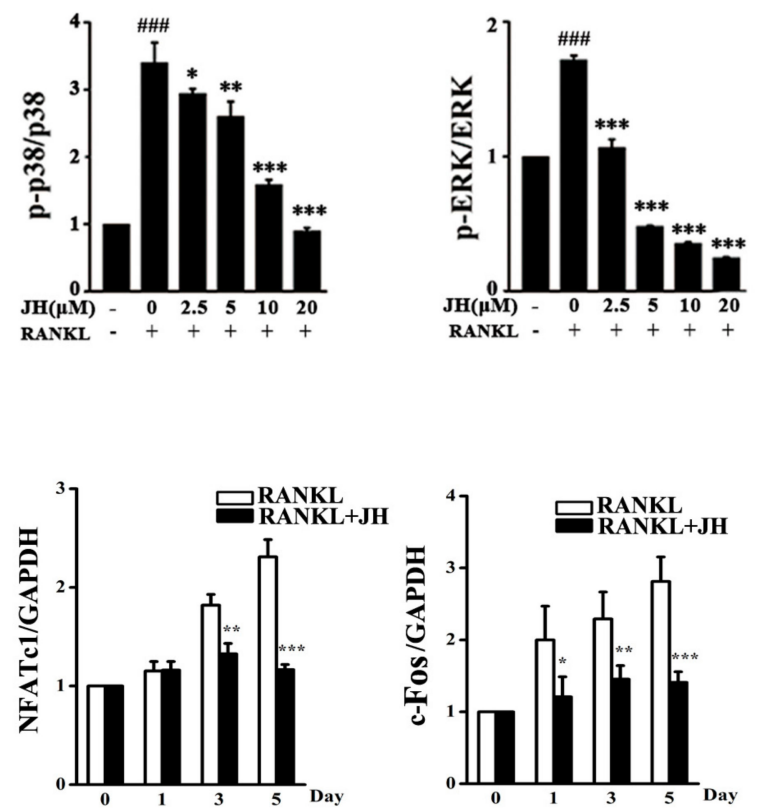

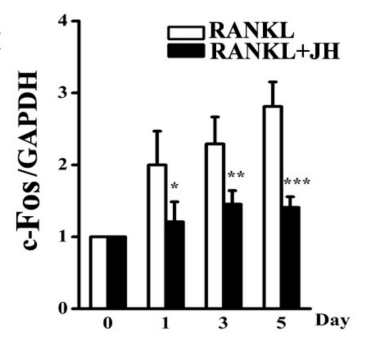

Figure 6. JH attenuates RANKL-stimulated activations of MAPKs (p38 and ERK). (A,C) After pretreatment with or without JH for $1 \mathrm{~h}, \mathrm{BMM}$ cells were stimulated with RANKL $\left(100 \mathrm{ng} \cdot \mathrm{mL}^{-1}\right.$ ) for $15 \mathrm{~min}$ in (A), and 0, 1, 3, 5 days in (C). Western blotting was performed to examine the levels of p-p38, total p38, p-ERK1/2, total ERK1/2, p-JNK, total JNK, IKB $\alpha$, NFATc1, c-Fos and GAPDH. $(\mathbf{B}, \mathbf{D})$ The ratios of p-p38/p38, p-ERK/ERK, NFATc1/GAPDH and c-Fos/GAPDH were quantified and calculated using ImageJ software $(n=3)$. All data were shown as mean $\pm \mathrm{SD}$; $p<0.05$, ** $p<0.01$ and ${ }^{* * *} p<0.001$ relative to RANKL-treated, JH-untreated controls. \#\# $p<0.001$ relative to RANKL-untreated, JH-untreated controls.

\section{Discussion}

To our best knowledge, the pharmacological studies of $\mathrm{JH}$ are very limited. Only few published papers have been found to report its bacteriostasis [16,17], tumor cell growth suppression $[19,20]$, and antidiabetic and antihyperlipidemic activities [21,22]. Most of these reported pharmacological properties have been characterized based on animal models, and their involvements in cellular and molecular mechanisms are still not determined. In this study, we found a novel pharmacological function of $\mathrm{JH}$, that is, it could effectively prevent wear particle-induced osteolysis in vivo and inhibit RANKL-induced osteoclastogenesis in vitro. And these inhibitory effects of $\mathrm{JH}$ might be mediated via the suppressed activation of MAPK (p38 and ERK).

In vivo studies showed JH significantly and dose-dependently suppressed Ti particle-induced bone destruction, as illustrated by significantly enhanced BMD and BV/TV. These results were further confirmed by histological analysis of tissues stained with H\&E and TRAP. The vehicle group showed significant increases in erosion area and number of TRAP-positive multinucleated osteoclasts, whereas these increases were effectively decreased by JH treatment, indicating that $\mathrm{JH}$ could exert a therapeutic effect to treat Ti particle-induced osteolysis through inhibiting osteoclast formation and function. 
Indeed, our in vitro results clearly demonstrated that $\mathrm{JH}$ could directly suppress the number and area of TRAP positive cells stimulated by RANKL. In addition, a significant decrease in the bone resorption activity was also observed in JH-treated groups on the basis of pit formation on bone slices, determined by a scanning electron microscope.

Having established that JH plays inhibitory roles in Ti particle-induced osteolysis model and RANKL-induced osteoclastogenesis, we further investigated the underlying molecular mechanisms. It is well known that RANKL interacts with its receptor RANK to induce the recruitment and activa-tion of an important adaptor protein, TRAF6. Activated TRAF6 complexes with TAK1-RACK-MKK6 or TAK-TABs to induce the activation of MAPK including p38, ERK and JNK. Also, TRAF6 can stimulate NF- $\mathrm{KB}$ activity either by interaction with $\mathrm{p} 62$ and $\alpha \mathrm{PKC}$ or via the regulation of TAK1-dependent phosphorylation [26]. Activated MAPK and NF- $k B$ have been reported to play key roles in RANKL-mediated osteoclastogenesis and function [25,27-31]. In our study, JH did not affect JNK and NF-KB signaling pathways, however, it could significantly suppress the phosphorylation levels of RANKL-mediated p38 and ERK. Interestingly, our previous study showed that, in synoviocyte cell line MH7A, JH also significantly inhibited TNF $\alpha$-induced p38 and ERK activation [23]. Thus, it is possible that blocking p 38 and ERK activation might be an important active site of JH. Certainly, further investigations are needed to unveil its direct binding sites.

Following activation of RANKL-stimulated MAPK signaling cascades, a series of downstream transcription factors are induced [31-33]. Among them, NFATc1 is considered the most important transcription factor required for the regulation of osteoclastogenesis. In this study, JH was demonstrated to significantly inhibit the induction of NFATc1, which was further supported by the decreased expression of c-fos and down-regulation of NFATc1-regulated osteoclastic marker genes, such as TRAP, CTR and CTSK by JH.

Throughout this study, JH was well-tolerated at the given doses without obvious drug toxicity to liver function in the treated mice. Additionally, our in vitro study showed that up to $160 \mu \mathrm{M}$ did not affect BMMs viability, suggesting that $\mathrm{JH}$ is a promising alternative medicine for osteoclast-related osteolytic therapy because of its efficiency and safety.

Despite these interesting findings, the present study has some limitations. Firstly, bone metabolism is a delicate balance of bone formation by osteoblasts and bone resorption by osteoclasts [34]. Bone formation has also been demonstrated to play an important role in osteolysis treatment $[35,36]$. It has been reported that, in addition to osteolysis, bone formation and repair of the osteolytic areas occur on the calvaria of Ti particle-induced osteolysis model [37]. In this study, the calvaria thickness in the vehicle group was observed to increase compared to that in the sham group, and this enlargement was gradually suppressed by JH treatment (Figure 2A). We speculated that the increased calvaria thickness was likely caused by bone formation and repair, and JH might play an inhibitory role in this process. Certainly, further investigations are needed to verify JH's action on osteoblasts and bone formation. Secondly, the main cause of clinical periprosthetic osteolysis is polyethylene rather than Ti particles [38]. However, Ti particles are well-characterized. Furthermore, both Ti and polyethylene have been reported to comparably activate osteoclast differentiation and bone resorption to induce osteolysis in vivo [39,40]. Thus, both animal models are suitable to be used for investigating the inhibitory action of $\mathrm{JH}$ on osteolysis. Thirdly, the murine calvarial model is a pathological model but not a disease model. The lack of implant and mechanical load, and a limited two-week duration of the osteolytic process should be considered in this study. Despite these limitations, our study clearly demonstrated that $\mathrm{JH}$ exerted inhibitory actions on osteoclast formation and function in vitro and in vivo, indicating that this compound may be a therapeutic candidate for treating osteolysis. 


\section{Materials and Methods}

\subsection{Animals}

A total of 28 healthy 8-week-old C57BL/ 6 mice were purchased from Beijing Vital River Laboratory Animal Technology Co. Ltd. (Beijing, China). They were housed under specific pathogen-free (SPF) conditions $\left(22^{\circ} \mathrm{C}, 12 \mathrm{~h} / 12 \mathrm{~h}\right.$ light/dark, 50-55\% humidity) and given free access to food and water. All the animal experiments were approval by the Experimental Committee of Nanjing Normal University (\#20160617, approved date: 7 June 2016).

\subsection{Media and Reagents}

Jatrorrhizine hydrochloride $\left(\mathrm{C}_{20} \mathrm{H}_{20} \mathrm{ClNO}_{4}\right.$, Purity $\left.\geq 98 \%\right)$ was purchased from Nanjing Zelang Medical Technology Co. Ltd. (Nanjing, Jiangsu, China). Alpha modified Minimal Essential Medium $(\alpha$-MEM) and fetal bovine serum (FBS) were purchased from Gibco (Gibco BRL, Grand Island, NY, USA). Recombinant mouse RANKL and M-CSF were provided by PeproTech (Rocky Hill, NJ, USA). MTS reagents were obtained from Sigma-Aldrich (St. Louis, MO, USA). TRIzol reagent was obtained from Invitrogen (Carlsbad, CA, USA). Primary anti-bodies targeting phosphorylated (p) ERK, p-p38, p-JNK1/2, ERK, p38, JNK1/2, I KB $\alpha$ and GAPDH were obtained from CST (Cell Signaling Technology, Inc., Beverly, MA, USA). Anti-NFATc1 and anti-c-fos antibodies were purchased from BD Biosciences (San Jose, CA, USA). Commercial kits for ALT and AST measurement were from Jiancheng Institute of Biotechnology (Nanjing, Jiangsu, China). Tartrate-resistant acid phosphatase (TRAP) staining kits were obtained from Sigma-Aldrich.

Titanium (Ti) particles (1 $3 \mu \mathrm{m}$ diameter) were purchased from Johnson Mathey Chemicals (Ward Hill, MA, USA). The particles were baked for $6 \mathrm{~h}$ at $180{ }^{\circ} \mathrm{C}$, and then treated with $70 \%$ ethanol for $48 \mathrm{~h}$. The sterile particles were stored in sterile PBS $\left(300 \mathrm{mg} \cdot \mathrm{mL}^{-1}\right)$ at $4{ }^{\circ} \mathrm{C}$ until required for use.

\subsection{Titanium Particle-Induced Calvarial Osteolysis Model}

To measure the osteolysis-suppressing effect of JH in vivo, we established a Ti particle-induced calvarial osteolysis model, as described by the previous studies [41,42]. Briefly, 28 male eight-week-old C57BL/ 6 mice were randomly divided into four groups $(n=7)$ : PBS control (sham), Ti particles in PBS (vehicle), Ti particles together with different doses of JH $\left(25 \mathrm{mg} \cdot \mathrm{kg}^{-1} \cdot \mathrm{day}^{-1}\right.$ and $100 \mathrm{mg} \cdot \mathrm{kg}^{-1} \cdot \mathrm{day}^{-1}$, respectively). The doses of $\mathrm{JH}$ were determined according to the previous studies [21,22] with modification from our preliminary experiments. The mice were anesthetized with avertin $\left(300 \mathrm{mg} \cdot \mathrm{kg}^{-1}\right.$ body weight), and the cranial periosteum was separated from the calvarium by sharp dissection. Thirty mg of Ti particles were then embedded under the periosteum at the middle suture of the calvaria. Two days after implantation, JH or PBS was intragastrically administrated every day for 14 days. At the end of the experiment, the mice were sacrificed and the serum was collected for ALT and AST analysis. And the calvaria were excised for micro-computerized tomography (CT) scanning and histological analysis.

\subsection{Serum ALT and AST Measurement}

Blood samples were harvested by heart puncture. After centrifugation, the supernatant was collected for ALT and AST measurement using the commercial kits according to the manufacturer's protocols.

\subsection{Micro-CT Scanning}

The calvaria were removed and fixed in $4 \%$ paraformaldehyde for $48 \mathrm{~h}$. After removal of the particles, the osteolysis in mouse calvariae was analyzed using a high-resolution micro-CT (SkyScan 1176, Bruker, Germany) with an isometric resolution of $9 \mu \mathrm{m}$. After reconstruction, we chosen 
a round region of interest (ROI) around the midline suture (4 mm diameter) for further quantitative analysis. BMD and BV/TV were measured using the CTan program (SkyScan, Kontich, Belgium).

\subsection{Histological and Histomorphometric Analysis}

After micro-CT scanning, the samples were decalcified, embedded, and stained with H\&E and TRAP. The bone area and the percentage of total porosity were calculated with Image Pro-Plus 5.0 software (Media Cybernetics, Silver Spring, MD, USA). The number of $\mathrm{TRAP}^{+}$osteoclasts were counted and normalized to bone area.

\subsection{Cell Viability Assay}

To examine the effect of JH on the viability of BMMs, a MTS assay was performed. BMMs were seeded in 96-well plates at a density of $6 \times 10^{3}$ cells/well and cultured overnight to adhere. Varying concentrations of JH were added to the cells and incubated for $48 \mathrm{~h}$. MTS/PMS mixture was then added and continuously cultured for another $4 \mathrm{~h}$ according to the manufacturer's instructions. Absorbance was measured at $490 \mathrm{~nm}$ using a microplate reader (BMG LABTECH GMBH, Ortenberg, Germany). The effect of JH on cell viability was expressed as percent cell viability with vehicle treated control cells set at $100 \%$.

\subsection{In Vitro Osteoclastogenesis Assay}

In vitro osteoclastogenesis assay was performed to examine the effect of $\mathrm{JH}$ on osteoclast differentiation. BMM cells were isolated, cultured and induced to differentiation as previously described [43]. Briefly, bone marrow cells isolated from femurs and tibias of 8-week-old C57BL/ 6 mice were incubated in complete cell culture media containing $30 \mathrm{ng} \cdot \mathrm{mL}^{-1} \mathrm{M}-\mathrm{CSF}$ in a T-75 $\mathrm{cm}^{2}$ flask for proliferation. When confluent, BMMs were seeded into 96-well plates at a density of $6000 \mathrm{BMMs} /$ well (in triplicate) and incubated overnight. BMMs were then treated with various concentrations of $\mathrm{JH}$ plus RANKL $\left(100 \mathrm{ng} \cdot \mathrm{mL}^{-1}\right)$ and M-CSF $\left(30 \mathrm{ng} \cdot \mathrm{mL}^{-1}\right)$. After five days, cells were fixed and stained for TRAP activity. TRAP $^{+}$multinucleated cells with more than three nuclei were counted as osteoclasts.

\subsection{Bone Resorption Pit Assay and F-Actin Ring Formation Assay}

Bone resorption assay was performed to test the effect of $\mathrm{JH}$ on osteoclast function. Bovine cortical bone slices $(100 \mu \mathrm{m})$ were cut using a cutting machine (IsoMet Low Speed Saw; Buehler Ltd., Lake Bluff, IL, USA). After sonication and extensive washing with MilliQ water, the bone slices were sterilized with $70 \%$ ethanol for $2 \mathrm{~min}$. The sterile bone slices were rinsed with sterile PBS for three times and then put into the 96 -well plates to incubate for $4 \mathrm{~h}$ with $\alpha$-MEM at $37{ }^{\circ} \mathrm{C}$ in $5 \% \mathrm{CO}_{2}$ atmosphere. After removal of the medium, BMM were seeded on the bone slices at a density of 8000 cells per well (in triplicate) and incubated with M-CSF $\left(30 \mathrm{ng} \cdot \mathrm{mL}^{-1}\right)$ and RANKL $\left(100 \mathrm{ng} \cdot \mathrm{mL}^{-1}\right)$ for $3-4$ days. When osteoclasts began to form, varying concentrations of $\mathrm{JH}$ were added and continuously cultured for another 2 days. The bone slices were then fixed with $4 \%$ paraformaldehyde and stained for TRAP activity. The number of TRAP positive multinucleated cells with more than three nuclei was counted. After gentle brushing the osteoclasts, the bone resorption pits were visualized by scanning electron microscopy (JSM 5610 LV; JEOL, Tokyo, Japan) and five view fields were randomly selected for each bone slice for further analysis. The areas of bone resorbed were determined with Image Pro-Plus 5.0 software (Media Cybernetics, Silver Spring, MD, USA). The result was shown as resorption area relative to the total area of bone. Experiments were repeated independently at least three times.

For F-actin ring formation assay, the experimental protocols for RANKL induction and JH treatment of BMM cells were consistent with those for bone resorption assay. Mature osteoclasts cultured on the bone slices were fixed with $4 \%$ paraformaldehyde for $15 \mathrm{~min}$ and then permeabilized with $0.5 \%$ Triton X-100 for $10 \mathrm{~min}$. F-actin rings were incubated with rhodamine-conjugated phalloidin for $15 \mathrm{~min}$ and nuclei with DAPI dye. Fluorescence images were acquired using a Nikon A1R 
resonance scanning confocal microscope (Nikon, Tokyo, Japan). The number and size of F-actin rings were analyzed using ImageJ software (NIH, Bethesda, MD, USA).

\subsection{Apoptosis Analysis by Flow Cytometry}

Apoptosis assay was performed using an Annexin V-FITC apoptosis detection kit (KenGEN Biotech. Co. Ltd., Nanjing, Jiangsu, China) according to the manufacturer's protocol. In brief, BMMs were exposed to $\mathrm{JH}(0,2.5,5,10 \mu \mathrm{m})$ for $24 \mathrm{~h}$ and then suspended in binding buffer. The cells were stained with Annexin V and propidium iodide (PI), and the results were analyzed with FACScan (Becton Dickinson, Franklin Lakes, NJ, USA) with the CellQuest program.

\subsection{RNA Isolation and Real-Time PCR Analysis}

BMMs were seeded in 12-well plates at a density of $4 \times 10^{4}$ cells/well. Cells were treated with different concentrations of $\mathrm{JH}(0,2.5,5,10 \mu \mathrm{m})$ for 5 days in the presence of M-CSF $\left(30 \mathrm{ng} \cdot \mathrm{mL}^{-1}\right)$ and RANKL (100 $\left.\mathrm{ng} \cdot \mathrm{mL}^{-1}\right)$. Total RNA was extracted and cDNA was synthesized from $500 \mathrm{ng}$ of total RNA using reverse transcriptase (TaKaRa Biotechnology Co., Ltd., Dalian, Shenyang, China). Real-time PCR was conducted using Mastercycler ep realplex 2 systems (Eppendorf, Hamburg, Germany) with a SYBR Premix Ex Tag kit (TaKaRa Biotechnology). Three independent experiments were conducted. And each experiment was performed in triplicate. The following real-time PCR primers were used: TRAP, 5'-CTGGAGTGCACGATGCCAGCGACA-3' (forward), 5' -TCCGTGCTCGGCGATGGACCAGA-3' (reverse); CTSK, 5'-CTTCCAATACGTGCAGCAGA-3' (forward), 5'-TCTTCAGGGCTTTCTCGTTC-3' (reverse); CTR, 5' -TCAGGAACCACGGAATCCTC-3' (forward), 5'-ACATTCAAGCGGATGCGTCT-3'

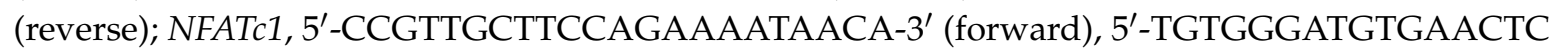
GGAA-3' (reverse); $c$-fos, $5^{\prime}$-CCAGTCAAGAGCATCAGCAA-3' (forward), 5'-AAGTAGTGCAGCCC GGAGTA-3' (reverse); $\beta$-actin, $5^{\prime}$-TCCTGTGGCATCCACGAAACT-3' (forward), 5'-GAAGCATTTG CGGTGGACGAT- $3^{\prime}$ (reverse). The relative expression of each target gene was normalized to $\beta$-actin $(n=3)$.

\subsection{Western Blot Analysis}

BMMs cells pre-treated with or without JH were washed twice with ice-cold PBS and then lysed with radioimmunoprecipitation assay (RIPA) buffer. After centrifugation, the protein in the supernatant was separated by SDS-PAGE electrophoresis, and then transferred to nitrocellulose membranes. After blocking with 5\% skim milk for $1 \mathrm{~h}$, the membranes were probed with specific antibodies against phospho-p38, phospho-ERK1/2, phospho-JNK1/2, p38, ERK1/2, JNK1/2, IKB, NFATc1, c-Fos and GAPDH. The antibody reactivity was detected using Enhanced Chemiluminescence (ECL) reagents (Amersham, Shanghai, China) according to manufacturer's instructions. Three independent experiments were conducted.

\subsection{Statistical Analysis}

The data from each experiment were presented as the mean \pm standard deviation (SD) from at least three independent experiments. Statistical comparisons were performed using one-way ANOVA, followed by Tukey's post hoc analysis. $p<0.05$ was considered statistically significant.

\section{Conclusions}

In summary, this study elucidated the inhibitory actions of $\mathrm{JH}$ on osteoclast formation and function in vitro and attenuated Ti particle-induced osteolysis in vivo. Further mechanism analysis revealed that the inhibition of JH was mediated via the suppression of MAPKs (p38 and ERK) signaling pathways. Our results strongly suggested that $\mathrm{JH}$ has a great potential to be developed into a novel therapeutic natural agent for treating periprosthetic osteolysis or other osteoclast-related osteolytic diseases. 
Author Contributions: M.L. designed the study. H.L., J.W., Q.S., G.C., S.S., X.M., H.Q., X.L. and L.X. performed the experiments. M.L. and H.L. analyzed the data. M.L. wrote the manuscript.

Funding: This study was supported by National Natural Science Foundation of China (Grant No. 31870895; 31171135), the Priority Academic Program Development of Jiangsu Higher Education Institutions (PAPD) and Top-notch Academic Programs Project of Jiangsu Higher Education Institutions (TAPP).

Conflicts of Interest: The authors declare no conflict of interest.

\section{References}

1. Harris, W.H. Wear and periprosthetic osteolysis: The problem. Clin. Orthop. 2001, 393, 66-70. [CrossRef]

2. Yunpeng, J.; Tanghong, J.; Wooley, P.H.; Shang-You, Y. Current research in the pathogenesis of aseptic implant loosening associated with particulate wear debris. Acta Orthop. Belg. 2013, 79, 1-9.

3. Gallo, J.; Vaculova, J.; Goodman, S.B.; Konttinen, Y.T.; Thyssen, J.P. Contributions of human tissue analysis to understanding the mechanisms of loosening and osteolysis in total hip replacement. Acta Biomater. 2014, 10, 2354-2366. [CrossRef] [PubMed]

4. Sundfeldt, M.; Carlsson, L.V.; Johansson, C.B.; Thomsen, P.; Gretzer, C. Aseptic loosening, not only a question of wear: A review of different theories. Acta Orthop. 2006, 77, 177-197. [CrossRef] [PubMed]

5. Holt, G.; Murnaghan, C.; Reilly, J.; Meek, R.M. The biology of aseptic osteolysis. Clin. Orthop. Relat. Res. 2007, 460, 240-252. [CrossRef] [PubMed]

6. Purdue, P.E.; Koulouvaris, P.; Potter, H.G.; Nestor, B.J.; Sculco, T.P. The cellular and molecular biology of periprosthetic osteolysis. Clin. Orthop. Relat. Res. 2007, 454, 251-261. [CrossRef] [PubMed]

7. Waddell, J.; Pritzker, K.P.; Boynton, E.L. Increased cytokine secretion in patients with failed implants compared with patients with primary implants. Clin. Orthop. Relat. Res. 2005, 434, 170-176. [CrossRef]

8. Weinstein, R.S.; Roberson, P.K.; Manolagas, S.C. Giant osteoclast formation and long-term oral bisphosphonate therapy. N. Engl. J. Med. 2009, 360, 1677-1678. [CrossRef] [PubMed]

9. Verron, E.; Bouler, J.M. Is bisphosphonate therapy compromised by the emergence of adverse bone disorders? Drug Discov. Today 2014, 19, 312-319. [CrossRef] [PubMed]

10. Gannon, S.C.; Cantley, M.D.; Haynes, D.R.; Hirsch, R.; Bartold, P.M. Azithromycin suppresses human osteoclast formation and activity in vitro. J. Cell Physiol. 2013, 228, 1098-1107. [CrossRef] [PubMed]

11. Olof, S.; Agata, R.; Thomas, E.; Mats, S.; Henrik, B.; Olle, M. Denosumab for treating periprosthetic osteolysis; study protocol for a randomized, double-blind, placebo-controlled trial. BMC Musculoskelet. Disord. 2016, 17, $1-10$.

12. Yamazaki, T.; Yamori, M.; Yamamoto, K.; Saito, K.; Asai, K.; Sumi, E.; Goto, K.; Takahashi, K.; Nakayama, T.; Bessho, K. Risk of osteomyelitis of the jaw induced by oral bisphosphonates in patients taking medications for osteoporosis: A hospital-based cohort study in Japan. Bone 2012, 51, 882-887. [CrossRef] [PubMed]

13. Mcclung, M.; Harris, S.T.; Miller, P.D.; Bauer, D.C.; Davison, K.S.; Dian, L.; Hanley, D.A.; Kendler, D.L.; Yuen, C.K.; Lewiecki, E.M. Bisphosphonate therapy for osteoporosis: Benefits, risks, and drug holiday. Am. J. Med. 2013, 126, 13-20. [CrossRef] [PubMed]

14. Marx, R.E. The deception and fallacies of sponsored randomized prospective double-blinded clinical trials: The bisphosphonate research example. Int. J. Oral. Maxillofac. Implant. 2014, 29, e37-e44. [CrossRef] [PubMed]

15. Hiligsmann, M.; Boonen, A.; Dirksen, C.D.; Sedrine, W.B.; Reginster, J.Y. Cost-effectiveness of denosumab in the treatment of postmenopausal osteoporotic women. Expert. Rev. Pharmacoecon. Outcomes Res. 2013, 13, 19-28. [CrossRef] [PubMed]

16. Slobodníková, L.; Kost'Álová, D.; Labudová, D.; Kotulová, D.; Kettmann, V. Antimicrobial activity of Mahonia aquifolium crude extract and its major isolated alkaloids. Phytother. Res. 2004, 18, 674-676. [CrossRef] [PubMed]

17. Volleková, A.; Košt'Álová, D.; Kettmann, V.; Tóth, J. Antifungal activity of Mahonia aquifolium extract and its major protoberberine alkaloids. Phytother Res. 2003, 17, 834-837. [CrossRef] [PubMed]

18. Chao, J.; Liao, J.W.; Peng, W.H.; Lee, M.S.; Pao, L.H.; Cheng, H.Y. Antioxidant, Analgesic, Anti-Inflammatory, and Hepatoprotective Effects of the Ethanol Extract of Mahonia oiwakensis Stem. Int. J. Mol. Sci. 2013, 14, 2928-2945. [CrossRef] [PubMed] 
19. Liu, R.; Cao, Z.; Pan, Y.; Zhang, G.; Yang, P.; Guo, P.; Zhou, Q. Jatrorrhizine hydrochloride inhibits the proliferation and neovascularization of C8161 metastatic melanoma cells. Anticancer Drugs 2013, 24, 667-676. [CrossRef] [PubMed]

20. Lin, C.C.; Ng, L.T.; Hsu, F.F.; Shieh, D.E.; Chiang, L.C. Cytotoxic effects of Coptis chinensis and Epimedium sagittatum extracts and their major constituents (berberine, coptisine and icariin) on hepatoma and leukaemia cell growth. Clin. Exp. Pharmacol. Physiol. 2004, 31, 65-69. [CrossRef] [PubMed]

21. Yan, F.U.; Ben-Rong, H.U.; Tang, Q.; Qin, F.U.; Zhang, Q.Y.; Xiang, J.Z. Effect of jatrorrhizine, berberine, Huanglian Decoction and compound-mimic prescription on blood glucose in mice. Chin. Tradit. Herb. Drugs 2005, 36, 548-551.

22. Yang, W.; She, L.; Yu, K.; Yan, S.; Zhang, X.; Tian, X.; Ma, S.; Zhang, X. Jatrorrhizine hydrochloride attenuates hyperlipidemia in a high-fat diet-induced obesity mouse model. Mol. Med. Rep. 2016, 14, 3277-3284. [CrossRef] [PubMed]

23. Qiu, H.; Sun, S.; Ma, X.; Cui, C.; Chen, G.; Liu, Z.; Li, H.; Liu, M. Jatrorrhizine Hydrochloride Suppresses Proliferation, Migration, and Secretion of Synoviocytes In Vitro and Ameliorates Rat Models of Rheumatoid Arthritis In Vivo. Int. J. Mol. Sci. 2018, 19, 1514. [CrossRef] [PubMed]

24. Takayanagi, H.; Kim, S.; Koga, T.; Nishina, H.; Isshiki, M.; Yoshida, H.; Saiura, A.; Isobe, M.; Yokochi, T.; Inoue, J.; et al. Induction and activation of the transcription factor NFATc1 (NFAT2) integrate RANKL signaling in terminal differentiation of osteoclasts. Dev. Cell 2002, 3, 889-901. [CrossRef]

25. Matsuo, K.; Galson, D.L.; Zhao, C.; Peng, L.; Laplace, C.; Wang, K.Z.; Bachler, M.A.; Amano, H.; Aburatani, H.; Ishikawa, H.; et al. Nuclear factor of activated T-cells (NFAT) rescues osteoclastogenesis in precursors lacking c-Fos. J. Biol. Chem. 2004, 279, 26475-26480. [CrossRef] [PubMed]

26. Jin, H.P.; Na, K.L.; Lee, S.Y. Current Understanding of RANK Signaling in Osteoclast Differentiation and Maturation. Mol. Cells 2017, 40, 706-713.

27. David, J.P.; Sabapathy, K.; Behrens, A.; Berger, B.; Hochedlinger, K.; Hoffmann, O.; Wagner, E.F. c-Jun phosphorylation by JNK1 but not by JNK2 is necessary for efficient osteoclastogenesis in response to TRANCE stimulation. J. Bone Miner. Res. 1999, 14, S149.

28. Ang, E.S.M.; Pavlos, N.J.; Chim, S.M.; Feng, H.T.; Scaife, R.M.; Steer, J.H.; Zheng, M.H.; Xu, J. Paclitaxel inhibits osteoclast formation and bone resorption via influencing mitotic cell cycle arrest and RANKL-induced activation of NF-KB and ERK. J. Cell. Biochem. 2012, 113, 946-955. [CrossRef] [PubMed]

29. Kang, J.H.; Lim, H.; Jeong, J.E.; Yim, M. Attenuation of RANKL-induced Osteoclast Formation via p38-mediated NFATc1 Signaling Pathways by Extract ofEuphorbia LathyrisL. J. Bone Metab. 2016, 23, 207-214. [CrossRef] [PubMed]

30. Soysa, N.S.; Alles, N.; Aoki, K.; Ohya, K. Osteoclast formation and differentiation: An overview. J. Med. Dent. Sci. 2016, 59, 65-74.

31. Xu, J.; Wu, H.; Ang, E.S.; Yip, K.; Woloszyn, M.; Zheng, M.; Tan, R. NF-кB modulators in osteolytic bone diseases. Cytokine Growth Factor Rev. 2009, 20, 7-17. [CrossRef] [PubMed]

32. Ikeda, F.; Nishimura, R.; Matsubara, T.; Tanaka, S.; Inoue, J.-I.; Reddy, S.V.; Hata, K.; Yamashita, K.; Hiraga, T.; Watanabe, T.; et al. Critical roles of c-Jun signaling in regulation of NFAT family and RANKL-regulated osteoclast differentiation. J. Clin. Investig. 2004, 114, 475-484. [CrossRef] [PubMed]

33. Takayanagi, H. New developments in osteoimmunology. Nat. Rev. Rheumatol. 2012, 8, 684-689. [CrossRef] [PubMed]

34. Kanno, Y.; Ishisaki, A.; Kawashita, E.; Chosa, N.; Nakajima, K.; Nishihara, T.; Toyoshima, K.; Okada, K.; Ueshima, S.; Matsushita, K.; et al. Plasminogen/Plasmin Modulates Bone Metabolism by Regulating the Osteoblast and Osteoclast Function. J. Biol. Chem. 2014, 289, 8952-8960. [CrossRef]

35. Wedemeyer, C.; Von, K.F.; Pingsmann, A.; Hilken, G.; Sprecher, C.; Saxler, G.; Henschke, F.; Löer, F.; Von, K.M. Stimulation of bone formation by zoledronic acid in particle-induced osteolysis. Biomaterials 2005, 26, 3719-3725. [CrossRef] [PubMed]

36. Córdova, L.A.; Trichet, V.; Escriou, V.; Rosset, P.; Amiaud, J.; Battaglia, S.; Charrier, C.; Berreur, M.; Brion, R.; Gouin, F.; et al. Inhibition of osteolysis and increase of bone formation after local administration of siRNA-targeting RANK in a polyethylene particle-induced osteolysis model. Acta Biomater. 2015, 13, 150-158. [CrossRef] [PubMed] 
37. Kaar, S.G.; Ragab, A.A.; Kaye, S.J.; Kilic, B.A.; Jinno, T.; Goldberg, V.M.; Bi, Y.; Stewart, M.C.; Carter, J.R.; Greenfield, E.M. Rapid repair of titanium particle-induced osteolysis is dramatically reduced in aged mice. J. Orthop. Res. 2001, 19, 171-178. [CrossRef]

38. Hirakawa, K.; Bauer, T.W.; Stulberg, B.N.; Wilde, A.H. Comparison and quantitation of wear debris of failed total hip and total knee arthroplasty. J. Biomed. Mater. Res. 1996, 31, 257-263. [CrossRef]

39. Von, K.M.; Jewison, D.E.; Sibonga, J.D.; Sprecher, C.; Morrey, B.F.; Loer, F.; Berry, D.J.; Scully, S.P. The effectiveness of polyethylene versus titanium particles in inducing osteolysis in vivo. J. Orthop. Res. 2004, 22, 237-243.

40. Taki, N.; Tatro, J.M.; Nalepka, J.L.; Togawa, D.; Goldberg, V.M.; Rimnac, C.M.; Greenfield, E.M. Polyethylene and titanium particles induce osteolysis by similar, lymphocyte-independent, mechanisms. J. Orthop. Res. 2005, 23, 376-383. [CrossRef] [PubMed]

41. Xiao, F.; Zhai, Z.; Jiang, C.; Liu, X.; Li, H.; Qu, X.; Ouyang, Z.; Fan, Q.; Tang, T.; Qin, A.; et al. Geraniin suppresses RANKL-induced osteoclastogenesis in vitro and ameliorates wear particle-induced osteolysis in mouse model. Exp. Cell Res. 2015, 330, 91-101. [CrossRef] [PubMed]

42. Wu, C.; Wang, W.; Tian, B.; Liu, X.; Qu, X.; Zhai, Z.; Li, H.; Liu, F.; Fan, Q.; Tang, T.; et al. Myricetin prevents titanium particle-induced osteolysis in vivo and inhibits RANKL-induced osteoclastogenesis in vitro. Biochem. Pharmacol. 2015, 93, 59-71. [CrossRef] [PubMed]

43. Liu, M.; Xu, L.; Ma, X.; Xu, J.; Wang, J.; Xian, M.; Zhou, X.; Wang, M.; Wang, F.; Qin, A.; et al. MAGED1 Is a Negative Regulator of Bone Remodeling in Mice. Am. J. Pathol. 2015, 185, 2653-2667. [CrossRef] [PubMed]

(C) 2018 by the authors. Licensee MDPI, Basel, Switzerland. This article is an open access article distributed under the terms and conditions of the Creative Commons Attribution (CC BY) license (http:/ / creativecommons.org/licenses/by/4.0/). 